\title{
TEM with in situ Ion Irradiation of Nuclear Materials: The IVEM-Tandem User Facility
}

\author{
Meimei Li ${ }^{1}$, Marquis A. Kirk ${ }^{1}$, Peter M. Baldo ${ }^{2}$ and Edward A. Ryan ${ }^{1}$ \\ 1. Nuclear Engineering Division, Argonne National Laboratory, Lemont, IL, USA \\ 2. Materials Science Division, Argonne National Laboratory, Lemont, IL, USA
}

The IVEM-Tandem User Facility at the Argonne National Laboratory (ANL) is a world-leading research facility for in situ TEM study of ion irradiation damage and ion implantation near the atomic resolution. The IVEM-Tandem Facility interfaces a $500 \mathrm{kV}$ ion implanter to a $300 \mathrm{kV}$ Hitachi H9000NAR transmission electron microscope. This combination allows experiments conducted with simultaneous ion irradiation/implantation and electron microscopy at temperatures ranging from 20 $1300 \mathrm{~K}$. With superior electron brightness of a LaB6 filament, the H-9000 microscope is ideally suited for imaging irradiation-induced defects using diffraction contrast, a well-established technique in most cases superior to other imaging techniques. This permits effective real time observation of nanoscale defect formation and evolution during irradiation. Coupled with well-controlled irradiation conditions (constant specimen orientation and area, specimen temperature, ion type, ion energy, dose rate, dose, and applied strain), it provides key information on structure and defect dynamics of a material in an irradiation environment.

The IVEM-Tandem Facility was commissioned in 1995 in the Materials Science Division of ANL, and was part of Argonne's Electron Microscopy Center, a National User Facility supported by the Department of Energy (DOE) Office of Science, Basic Energy Science. It was recently transitioned to the Nuclear Engineering Division of ANL, currently supported by the DOE Office of Nuclear Energy. The IVEM-Tandem serves more than 20 active user groups from universities, national laboratories, and nuclear industry in the U.S. and abroad, conducting research in areas of advanced alloys, accident tolerant materials and fuels, radiation-resistant fuel cladding, storage materials for spent fuels, and validation and verification of computer modeling and simulations. A facility upgrade is being considered to best address the critical problems of fission and fusion energy materials.

TEM with in situ ion irradiation has made tremendous contributions to the understanding of defect production, accumulation and evolution under irradiation. Many of fundamental questions of radiation damage, e.g. single cascades, cascade - cascade, or cascade - subcascade interactions, defect production and annihilation rates of visible defect clusters, defect and dislocation interactions, etc. have been answered. A recent new direction is to predict neutron damage in bulk through in situ ion irradiation studies of thin films and coordinated computer modeling so that neutron irradiation effects can be evaluate by easily controlled ion irradiation experiments. For example, TEM with in situ ion irradiation experiments was conducted on molybdenum with $1 \mathrm{MeV} \mathrm{Kr}$ ions at the IVEM-Tandem Facility in combination with rate theory modeling. The in situ ion irradiation experiments were explicitly designed to compare with neutron irradiation data of the identical material reported in a previous study [1]. A spatially-dependent cluster dynamic model was developed to explicitly simulate the damage by $1 \mathrm{MeV}$ $\mathrm{Kr}$ ion irradiation in a Mo thin film with temporal and spatial dependence of defect distribution. Both in situ ion irradiation and neutron irradiation produced the same defect structure (i.e. dislocation loops) in Mo irradiated to low-doses at $80^{\circ} \mathrm{C}$. This allowed direct, quantitative comparisons of defect number 
densities and size distributions. In situ ion irradiation experiments took advantage of thin foil specimens, and used the thickness of a thin foil as an important variable in describing the defect depth distribution by three-dimensional diffraction contrast electron tomography. This additional spatial dimension improves the rate theory based cluster dynamic model for defect reaction kinetics and enabled direct comparison with experiments of defect structures at different foil depths and times (doses) [2,3]. The experimentally-validated damage model of ion-irradiated thin films was then used to predict neutron damage in bulk materials and validated by the neutron irradiation data. This study demonstrates a promising new direction in understanding and predicting neutron damage in bulk with in situ ion irradiation of thin films closely coupled with computer modeling using the IVEM-Tandem Facility.

\section{References:}

[1] Meimei Li, M. Eldrup, T. S. Byun, N. Hashimoto, L. L. Snead, S. J. Zinkle, J. Nucl. Mater. 376 (2008) 11.

[2] Meimei Li, M.A. Kirk, P.M. Baldo, Donghua Xu, and B. D. Wirth, Phil Mag. 92 (2012) 2048. [3] D. Xu, B. D. Wirth, M. Li, and M. Kirk, Acta Mater. 60 (2012) 4286.

[4] The electron microscopy with in situ ion irradiation was accomplished at the Argonne National Laboratory at the IVEM-Tandem Facility, a user facility funded by the Department of Energy Office of Nuclear Energy, operated under Contract No. DE-AC02-06CH11357 by UChicago Argonne, LLC. 